\title{
The Free Energy of the Quantum Heisenberg Ferromagnet at Large Spin
}

\author{
M. Correggi, A. Giuliani \\ Dipartimento di Matematica, Università degli Studi Roma Tre, \\ L.go S. Leonardo Murialdo 1, 00146, Rome, Italy.
}

November 5, 2018

\begin{abstract}
We consider the spin- $S$ ferromagnetic Heisenberg model in three dimensions, in the absence of an external field. Spin wave theory suggests that in a suitable temperature regime the system behaves effectively as a system of non-interacting bosons (magnons). We prove this fact at the level of the specific free energy: if $S \rightarrow \infty$ and the inverse temperature $\beta \rightarrow 0$ in such a way that $\beta S$ stays constant, we rigorously show that the free energy per unit volume converges to the one suggested by spin wave theory. The proof is based on the localization of the system in small boxes and on upper and lower bounds on the local free energy, and it also provides explicit error bounds on the remainder.
\end{abstract}

\section{Introduction}

An important open problem in theoretical and mathematical physics is the proof of long range order in the three-dimensional (3D) quantum Heisenberg ferromagnet (FM) at low temperatures. While the existence of long range order at low temperatures in the classical Heisenberg model and in the quantum Heisenberg antiferromagnet can be proved by reflection positivity methods [FSS, DLS, the broken phase of the quantum ferromagnet eluded any rigorous treatment so far.

From a heuristic point of view, a very useful and suggestive representation of the quantum FM is in terms of spin waves, an idea first introduced by Bloch in his seminal work B1, B2]. The spin waves are the lowest energy excitations, which give the dominant contribution to the free energy at low temperatures; they satisfy a Bose statistics and are in many respects the analogues of the phonons in crystals (see, e.g., $\mathrm{Ke}$ for a classical and comprehensive review). Bloch's theory was later generalized in several directions by Herring and Kittel [HK], Holstein and Primakoff [HP], Dyson [D1, and it was used, among other things, to compute a low temperature expansion for the spontaneous magnetization in zero external magnetic field: after a few erroneous attempts $\mathrm{Kr}, \mathrm{Op}, \mathrm{Sc}, \mathrm{VK}$, Dyson's result $\mathrm{D} 2$ was confirmed by a number of different methods $\mathrm{CH}, \mathrm{KL}, \mathrm{M}, \mathrm{Og}, \mathrm{RL},[\mathrm{Sz}, \mathrm{VLP}, \mathrm{W}, \mathrm{YW}, \mathrm{Z}]$ and further extended, more recently, by the effective Lagrangian method $\mathrm{H1}$, H2]. The conclusion is that at low energies the corrections to the simple Bloch's theory coming from the interactions among spin waves are so small that for most practical purposes the linear theory is enough, both in the presence or in the absence of an external magnetic field.

While physically Bloch's theory is accepted and in good agreement with experiments, from a more mathematical point of view there is no confirmation of its correctness yet. It is fair to say that the current mathematical methods of quantum many body systems are still far from allowing us to prove the existence of a spontaneous magnetization in the quantum Heisenberg ferromagnet and to possibly confirm the exactness of Dyson's computation D2.

An easier, but still unsolved problem is to prove the correctness of spin wave theory at the level of thermodynamic rather than correlation functions, at least in the large spin limit, where the quantum 
FM is (formally) close to its classical counterpart L or to the free Bose gas HP vHBW], depending on the temperature regime which we look the system at. Some progress in this direction is due to Conlon and Solovej CS1, who proved that the free energy of the quantum Heisenberg FM in the presence of a large magnetic field is the same as the one of a free Bose gas, asymptotically for large onsite spin $S$. For small (i.e., 1/2) spin and small temperatures, Conlon and Solovej [CS2] and Toth [T] derived an upper bound on the free energy, close but not exactly equal to Bloch's theory prediction. In the large spin limit, it was unclear whether the rigorous justification of spin wave theory by Conlon and Solovej CS1] could be extended to the case of zero magnetic field, which is much more subtle and unstable with respect to temperature-induced excitations, due to the global rotational symmetry of the model. For the classical counterpart of this problem, that is the convergence of the large-S classical Heisenberg model to the gaussian one, Conlon and Solovej managed to show the uniformity of their results as the magnetic field is taken to zero CS3 (an even stronger convergence result to the gaussian model for the case of the classical rotator model is due to Bricmont et al [BFLLS]). However, their proof did not apply to the quantum case, see the comments after [CS3, Theorem 2.1]. The large spin limit for the XXZ chain in the absence of a magnetic spin was studied by Michoel and Nachtergaele MN1, MN2], but their proof required the presence of an energy gap in the spectrum of the spin chain.

In this paper, we solve the issue by extending these results to the 3D quantum rotationally invariant (gapless) case; namely, we show that the free energy of the 3D quantum Heisenberg FM at temperatures of order $S$ converges as $S \rightarrow \infty$ to the one of the non-interacting magnon gas. This rigorously proves (in a weak sense) the validity of the spin wave approximation at leading order in $S$ for $S$ large. We use the Holstein-Primakoff representation of the quantum Heisenberg FM in terms of interacting bosons, and we take advantage of the methods and the ideas recently developed by Lieb, Seiringer and Yngvason to treat the interacting Bose gas at low densities [LS, LSY] LY] (see [LSSY] for a review): key ingredients of our proof are: (i) a localization bound, allowing us to coarse grain the system in finite boxes; (ii) a priori bounds on the local energy, which make use of the energy gap in the box and allow us to drop the states with total spin far from the maximum.

The paper is organized as follows. In Section 1.1 we define the model and state our main results. In Section 1.2 we review the Holstein-Primakoff representation and briefly review spin wave theory. In Section 2 we prove our main theorem by deriving suitable lower (Section 2.1) and upper (Section 2.2. bounds.

\subsection{The Model and the Main Result}

We consider a ferromagnetic Heisenberg model with nearest neighbor interactions which is associated with the Hamiltonian 1

$$
H_{\Lambda}^{\text {per }}:=J \sum_{\langle\mathbf{x}, \mathbf{y}\rangle \subset \Lambda}\left(S^{2}-\hat{\mathbf{S}}_{\mathbf{x}} \cdot \hat{\mathbf{S}}_{\mathbf{y}}\right)
$$

where $J>0, \Lambda$ is a cubic portion of side length $L$ of $\mathbb{Z}^{3}$ with periodic boundary conditions, the sum runs over the nearest neighbor pairs in $\Lambda$, and $\hat{\mathbf{S}}_{\mathbf{x}}$ is a three-components spin $S$ operator. This means that the components of $\hat{\mathbf{S}}_{\mathbf{x}}$, denoted by $\hat{S}_{\mathbf{x}}^{j}, j=1,2,3$, satisfy:

$$
\left[\hat{S}_{\mathbf{x}}^{j}, \hat{S}_{\mathbf{y}}^{k}\right]=i \varepsilon_{j k l} \hat{S}_{\mathbf{x}}^{l} \delta_{\mathbf{x}, \mathbf{y}}, \quad \hat{\mathbf{S}}_{\mathbf{x}}^{2}=\left(\hat{S}_{\mathbf{x}}^{1}\right)^{2}+\left(\hat{S}_{\mathbf{x}}^{2}\right)^{2}+\left(\hat{S}_{\mathbf{x}}^{3}\right)^{2}=S(S+1),
$$

where in the first equation $\varepsilon_{k l m}$ is the completely antisymmetric symbol, while in the second equation $S$ is the size of the spin, with $2 S$ an integer. We shall denote by $\mathscr{H}_{\Lambda}$ the Hilbert space of spin configurations in $\Lambda$ such that $\hat{\mathbf{S}}_{\mathbf{x}}^{2}=S_{\mathbf{x}}\left(S_{\mathbf{x}}+1\right)$ with $S_{\mathbf{x}}=S$; a convenient basis for this space is $\left|\left\{S_{\mathbf{x}}^{3}\right\}\right\rangle:=\bigotimes_{\mathbf{x} \in \Lambda}\left|S_{\mathbf{x}}^{3}\right\rangle$, with $-S \leq S_{\mathbf{x}}^{3} \leq S, \forall \mathbf{x} \in \Lambda$. Note that the Hamiltonian (1.1) is normalized in such a way that the ground state energy is zero.

Our main object of interest is the free-energy per site

$$
f(S, \beta, \Lambda):=-\frac{1}{\beta|\Lambda|} \log \mathcal{Z}(S, \beta, \Lambda), \quad \text { with } \quad \mathcal{Z}(S, \beta, \Lambda)=\operatorname{Tr}_{\mathscr{H}_{\Lambda}}\left(\exp \left\{-\beta H_{\Lambda}^{\text {per }}\right\}\right),
$$

\footnotetext{
${ }^{1}$ We use the convention of distinguishing (when needed) between operators and numbers or eigenvalues by means of a on top of the first ones. As usual we also denote vectors by bold letters.
} 
and the thermodynamic limit of the specific free energy, namely, $f(S, \beta):=\lim _{\Lambda \rightarrow \infty} f(S, \beta, \Lambda)$. Our goal is to compute $f(S, \beta)$ to the leading order in $S$ for $S$ large in a suitable temperature regime (to be discussed below) and to prove that it coincides within explicitly estimated errors with the free energy of a free Bose gas. More precisely, our main result, which generalizes a similar result in CS1, Theorem 4.2] proven for a non-zero (in fact huge, i.e., $h=\mathcal{O}(S)$ ) magnetic field, can be stated as follows.

Theorem 1.1 (Free energy asymptotics).

Assume that $\beta=\tilde{\beta} S^{-1}$ as $S \rightarrow \infty$ for some $\tilde{\beta}>0$ constant independent of $S$. Then one has

$$
\frac{f(S, \beta)}{S}=\frac{1}{\tilde{\beta}} \int_{\mathcal{B}} \frac{\mathrm{d} \mathbf{k}}{(2 \pi)^{3}} \log \left(1-e^{-\tilde{\beta} J \varepsilon(\mathbf{k})}\right)+\mathcal{O}\left(S^{-1 / 8}(\log S)^{1 / 4}\right),
$$

where $\varepsilon(\mathbf{k}):=\sum_{i=1}^{3}\left(1-\cos k_{i}\right)$ is the dispersion relation and $\mathcal{B}=[-\pi, \pi]^{3}$ is the first Brillouin zone.

Remark 1.1 (Zero temperature limit)

By a direct inspection of the proof, it is easy to verify that all the estimates are uniform in $\tilde{\beta}>0$, if $\tilde{\beta}$ is well separated from 0 . Hence the result can be straightforwardly extended to any $\tilde{\beta}=\tilde{\beta}(S)$ function of $S$, provided that $\liminf _{S \rightarrow \infty} \tilde{\beta}(S)>0$, including the case that $\lim _{S \rightarrow \infty} \tilde{\beta}=\infty$. This last case for the spin-1/2 Heisenberg ferromagnet was considered in CS2] and [T], where only upper bounds on the free energy were proven.

Remark 1.2 (External magnetic field)

The addition of an external magnetic field would not affect the result proven in the Theorem above. In fact, as already mentioned, the case of a magnetic field $h$ of order $\mathcal{O}(S)$ was already considered in CS1. However any magnetic field $h \ll S$ would produce a contribution to the r.h.s. of (1.4) of order $h / S$ and thus would not affect the leading order term, irrespective of the sign of $h$.

The intuition behind this result is based on a well-known bosonic representation of the Heisenberg model, first proposed by Holstein and Primakoff in [HP], reviewed in the following section.

\subsection{Bose Gas Representation and Magnon Approximation}

It is well known since the pioneering work Holstain-Primakoff $\mathrm{HP}$ that the Heisenberg Hamiltonian can be rewritten in terms of suitable creation and annihilation operators, so that the excitations of the model can be described as Bose particles exactly as phonons in crystals. For any $\mathbf{x} \in \Lambda$ we set

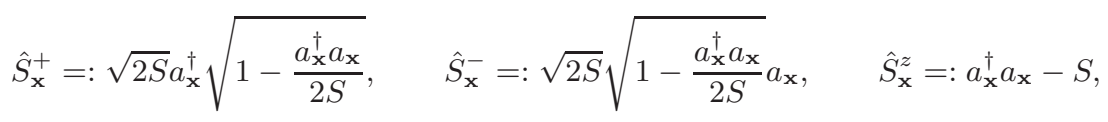

where $a_{\mathbf{x}}^{\dagger}, a_{\mathbf{x}}$ are bosonic creation and annihilation operators. We can associate with such Bose modes an Hilbert space $\mathscr{F}_{\Lambda}$ isomorphic to the spin Hilbert space $\mathscr{H}_{\Lambda}$ by diagonalizing the number of particles operator $\hat{n}_{\mathbf{x}}:=a_{\mathbf{x}}^{\dagger} a_{\mathrm{x}}$ and we denote such a basis by $\left.\left.\mid\left\{n_{\mathbf{x}}\right\}\right):=\bigotimes_{\mathbf{x} \in \Lambda} \mid n_{\mathbf{x}}\right)$. Note that the constraint $-S \leq S_{\mathrm{x}}^{z} \leq S$ translates into the requirement

$$
n_{\mathrm{x}} \leq 2 S
$$

i.e., the Hilbert space is truncated and the occupation number per site can not exceed $2 S$. The isomorphism between the Hilbert spaces $\mathscr{H}_{\Lambda}$ and $\mathscr{F}_{\Lambda}$ can be implemented by means of the one-to-one correspondence $\left.\mid n_{\mathrm{x}}\right) \longleftrightarrow\left|S_{\mathrm{x}}^{3}=n_{\mathrm{x}}-S\right\rangle$, so that, for instance, the ground state with all the spins pointing down is $\left.\mid\left\{n_{\mathrm{x}}=0\right\}\right)$.

The Hamiltonian $H_{\Lambda}^{\text {per }}$ can be rewritten in terms of the creation and annihilation operators as

$$
\mathcal{H}_{\Lambda}^{p e r}=J S \sum_{\substack{\mathbf{x}, \mathbf{y} \in \Lambda \\\|\mathbf{x}-\mathbf{y}\|_{\Lambda}=1}}\left\{-a_{\mathbf{x}}^{\dagger}\left(1-\frac{a_{\mathbf{x}}^{\dagger} a_{\mathbf{x}}}{2 S}\right)^{1 / 2}\left(1-\frac{a_{\mathbf{y}}^{\dagger} a_{\mathbf{y}}}{2 S}\right)^{1 / 2} a_{\mathbf{y}}+a_{\mathbf{x}}^{\dagger} a_{\mathbf{x}}-\frac{1}{2 S} a_{\mathbf{x}}^{\dagger} a_{\mathbf{y}}^{\dagger} a_{\mathbf{x}} a_{\mathbf{y}}\right\},
$$


where $\|\cdot\|$ is the euclidean distance on the torus $\Lambda$. For $S$ large, this representation naturally leads to a decomposition of $\mathcal{H}_{\Lambda}^{\text {per }}$ into "free" and "interacting" parts:

$$
\mathcal{H}_{\Lambda}^{\text {per }}=\mathcal{H}_{0, \Lambda}^{\text {per }}+\mathcal{K}_{\Lambda}^{\text {per }}, \quad \mathcal{H}_{0, \Lambda}^{\text {per }}:=S J \sum_{\substack{\mathbf{x}, \mathbf{y} \in \Lambda \\\|\mathbf{x}-\mathbf{y}\|_{\Lambda}=1}} a_{\mathbf{x}}^{\dagger}\left(a_{\mathbf{x}}-a_{\mathbf{y}}\right),
$$

where $\mathcal{K}_{\Lambda}^{\text {per }}$ is at least quartic in the Bose operators and is formally of relative size $1 / S$ with respect to the quadratic part. The magnon approximation simply amounts to drop the interaction term $\mathcal{K}_{\Lambda}^{\text {per }}$ from the energy $\mathcal{H}_{\Lambda}^{\text {per }}$ and study the free Bose gas so obtained. By means of the Fourier transform the free Hamiltonian $\mathcal{H}_{0, \Lambda}^{\text {per }}$ can be easily diagonalized: given any $\mathbf{k} \in \Lambda^{*}$, where $\Lambda^{*}$ denotes the dual lattice, i.e., $\mathbf{k}=\frac{2 \pi}{L} \mathbf{m}$, with $0 \leq m_{i}<L$, we set $\varepsilon(\mathbf{k}):=\sum_{i=1}^{3}\left(1-\cos k_{i}\right)$ and define the creation and annihilation operators for the Fourier modes as $\tilde{a}_{\mathbf{k}}:=L^{-3 / 2} \sum_{\mathbf{x} \in \Lambda} \exp \{i \mathbf{k} \cdot \mathbf{x}\} a_{\mathbf{x}}$, so that the Hamiltonian (1.8) can be rewritten $\mathcal{H}_{0, \Lambda}^{p e r}=S J \sum_{\mathbf{k} \in \Lambda^{*}} \varepsilon(\mathbf{k}) \tilde{a}_{\mathbf{k}}^{\dagger} \tilde{a}_{\mathbf{k}}$.

The free energy of such a Bose gas should be computed by taking the trace of $\exp \left\{-\beta \mathcal{H}_{0, \Lambda}^{\text {per }}\right\}$ on the truncated Hilbert space $\mathscr{F}_{\Lambda}$, where the occupation number of each site cannot exceed $2 S$. However, in the large $S$ limit one expects that such a constraint can be removed in the calculation of the free-energy, up to higher order corrections, and in the thermodynamic limit this would yield exactly the dominant contribution in the r.h.s. of (1.4).

\section{Proofs}

In this section we prove Theorem 1.1. The asymptotics (1.4) is obtained by comparing suitable upper (Section 2.2) and lower (Section 2.1) bounds to the free energy of the ferromagnetic Heisenberg model.

\section{$2.1 \quad$ Lower Bound}

Here we prove the lower bound to the free energy. The main ingredients in the proof are a localization of the energy into Neumann boxes and two simple lower bounds on the localized Hamiltonian: the first one uses the energy gap in the box to show that the states with total spin far from the maximum have large energy; the second one is a rough bound on the contribution from the bosonic interaction part, which will be useful only after having restricted the trace to states with third component of the total spin close to the minimum value (the proofs of these two estimates are deferred to Appendix A).

Proposition 2.1 (Free energy lower bound).

Assume that $\beta=\tilde{\beta} S^{-1}$ as $S \rightarrow \infty$ for some $\tilde{\beta}>0$ constant. Then one has

$$
\frac{f(S, \beta)}{S} \geq \frac{1}{\tilde{\beta}} \int_{\mathcal{B}} \frac{\mathrm{d} \mathbf{k}}{(2 \pi)^{3}} \log \left(1-e^{-\tilde{\beta} J \varepsilon(\mathbf{k})}\right)-\mathcal{O}\left(S^{-1 / 8}(\log S)^{1 / 4}\right) .
$$

Proof. The first step towards the proof of (2.1) is a localization of the energy into small boxes of side length $\ell$ with Neumann conditions at the boundary. We partition the big box $\Lambda$ into boxes $\Lambda_{i}$ of side $\ell$ and use the positivity of the bond energy for all the bonds connecting a site in $\Lambda_{i}$ with a site in $\Lambda_{j}, i \neq j$. Correspondingly, we bound the original Hamiltonian $H$ from below as $H \geq \sum_{i} H_{\Lambda_{i}}^{N}$ where $H_{\Lambda_{i}}^{N}$ depends only on the degrees of freedom associated with the spins in $\Lambda_{i}$ and has free conditions at the boundary:

$$
H_{\Lambda_{i}}^{N}=\frac{J}{2} \sum_{\substack{\mathbf{x}, \mathbf{y} \in \Lambda_{i} \\|\mathbf{x}-\mathbf{y}|=1}}\left(S^{2}-\hat{\mathbf{S}}_{\mathbf{x}} \cdot \hat{\mathbf{S}}_{\mathbf{y}}\right)
$$

where $|\cdot|$ is the euclidean distance on $\mathbb{Z}^{3}$. The Hilbert space on which $H_{\Lambda_{i}}^{N}$ is assume to act (i.e., the restriction of $\mathscr{H}_{\Lambda}$ to $\Lambda_{i}$ ) will be denoted by $\mathscr{H}_{\Lambda_{i}}$.

Obviously, all the Hamiltonians $H_{\Lambda_{i}}^{N}$ commute among each other. Therefore,

$$
\mathcal{Z}(S, \beta, \Lambda) \leq\left[\operatorname{Tr}_{\mathscr{H}_{\Lambda_{1}}}\left(\exp \left\{-\beta H_{\Lambda_{1}}^{N}\right\}\right)\right]^{L^{3} / \ell^{3}}=: \mathcal{Z}^{N}\left(S, \beta, \Lambda_{1}\right)^{L^{3} / \ell^{3}}
$$


where the trace $\operatorname{Tr}_{\mathscr{H}_{\Lambda_{1}}}$ is only over the spin degrees of freedom within $\Lambda_{1}$.

In the computation of $\operatorname{Tr} \mathscr{H}_{\Lambda_{1}} \exp \left\{-\beta H_{\Lambda_{1}}^{N}\right\}$ we now distinguish between the states with total spin close to the maximum, from those with "small spin", which have a big energy and, thus, give a small contribution to the free energy. Note that the Hamiltonian $H_{\Lambda_{1}}^{N}$ is invariant under global rotations, that is $H_{\Lambda_{1}}^{N}$ commutes with the three components of the total spin $\hat{\mathbf{S}}_{T}:=\sum_{\mathbf{x} \in \Lambda_{1}} \hat{\mathbf{S}}_{\mathbf{x}}$ in $\Lambda_{1}$. Therefore, $H_{\Lambda_{1}}^{N}$ is block diagonal with respect to the decomposition $\mathscr{H}_{\Lambda_{1}}=\bigoplus_{S_{T}=0}^{S \ell^{3}} \bigoplus_{S_{T}^{3}=-S_{T}}^{S_{T}} \mathscr{H}_{S_{T}, S_{T}^{3}}$, where $\mathscr{H}_{S_{T}, S_{T}^{3}}$ is the subspace of $\mathscr{H}_{\Lambda_{1}}$ on which $\hat{\mathbf{S}}_{T}^{2}=S_{T}\left(S_{T}+1\right)$ and $\hat{S}_{T}^{3}=S_{T}^{3}$. On each $\mathscr{H}_{S_{T}, S_{T}^{3}}$, the Hamiltonian can be bounded from below as follows, independently of the value of $S_{T}^{3}$ (see Appendix A for a proof).

Proposition 2.2 (Lower bound on $H_{\Lambda_{1}}^{N}$ ).

There exists a positive constant $c>0$ such that, if $S-\ell^{-3} S_{T}>3 J c^{-1} \ell^{2}$, then

$$
\left.H_{\Lambda_{1}}^{N}\right|_{\mathscr{H}_{S_{T}, S_{T}^{3}}} \geq c \ell S\left(S-\frac{S_{T}}{\ell^{3}}\right) .
$$

We now split the trace of interest in two parts:

$$
\mathcal{Z}^{N}\left(\beta, S, \Lambda_{1}\right)=\sum_{S_{T} \geq S_{\star} \ell^{3}} \sum_{S_{T}^{3}=-S_{T}}^{S_{T}} \operatorname{Tr}_{\mathscr{H}_{S_{T}, S_{T}^{3}}} \exp \left\{-\beta H_{\Lambda_{1}}^{N}\right\}+\mathcal{R},
$$

where $S_{\star}<S$ is a parameter which is going to be fixed later, and the rest $\mathcal{R}$ can be bounded by means of (2.4) as follows:

$$
\mathcal{R}=\sum_{S_{T}<S_{\star} \ell^{3}} \sum_{S_{T}^{3}=-S_{T}}^{S_{T}} \operatorname{Tr}_{\mathscr{H}_{S_{T}, S_{T}^{3}}} \exp \left\{-\beta H_{\Lambda_{1}}^{N}\right\} \leq(2 S+1)^{\ell^{3}} e^{-c \tilde{\beta} \ell S\left(1-\frac{S_{\star}}{S}\right)},
$$

provided that $S-S_{*}>3 J c^{-1} \ell^{2}$, which can be satisfied by picking

$$
\frac{S_{\star}}{S}=1-\frac{2 \ell^{2} \log (2 S+1)}{c \tilde{\beta} S}
$$

and $\ell, S$ large enough, so that $\mathcal{R} \leq(2 S+1)^{-\ell^{3}} \ll \mathcal{O}(1)$.

We are now left with the main contribution to the trace (the one involving $S_{T} \geq S_{*} \ell^{3}$ ), to be called $\tilde{\mathcal{Z}}\left(\beta, S, \Lambda_{1}\right)$. By using once again the fact that $H_{\Lambda_{1}}^{N}$ commutes with $\hat{\mathbf{S}}_{T}$, we find that $\operatorname{Tr}_{\mathscr{H}_{S_{T}, S_{T}^{3}}} \exp \left\{-\beta H_{\Lambda_{1}}^{N}\right\}$ is independen ${ }^{2}$ of $S_{T}^{3}$, so that

$$
\tilde{\mathcal{Z}}\left(\beta, S, \Lambda_{1}\right)=\sum_{S_{T}=S_{\star} \ell^{3}}^{S \ell^{3}}\left(2 S_{T}+1\right) \operatorname{Tr}_{\mathscr{H}_{S_{T},-S_{T}}} \exp \left\{-\beta H_{\Lambda_{1}}^{N}\right\} .
$$

We can now apply the boson representation given in Eq. (1.5), which implies

$$
\tilde{\mathcal{Z}}\left(\beta, S, \Lambda_{1}\right) \leq\left(2 S \ell^{3}+1\right) \sum_{\substack{\left\{n_{\mathbf{x}}\right\}, \mathbf{x} \in \Lambda_{1} \\ \sum n_{\mathbf{x}} \leq\left(S-S_{\star}\right) \ell^{3}}}\left(\left\{n_{\mathbf{x}}\right\}\left|\exp \left\{-\beta \mathcal{H}_{\Lambda_{1}}^{N}\right\}\right|\left\{n_{\mathbf{x}}\right\}\right)
$$

\footnotetext{
2 This can be proved as follows. Let us indicate by $\mathbf{x}_{1}, \ldots, \mathbf{x}_{\ell^{3}}$ the sites of $\Lambda_{1}$ labeled in lexicographic order. By the theory of the composition of angular momenta, a bona fide basis for $\mathscr{H}_{\Lambda_{1}}$ is provided by the common eigenvectors of $\left(\hat{\mathbf{S}}_{\mathbf{x}_{1}}+\hat{\mathbf{S}}_{\mathbf{x}_{2}}\right)^{2},\left(\hat{\mathbf{S}}_{\mathbf{x}_{1}}+\hat{\mathbf{S}}_{\mathbf{x}_{2}}+\hat{\mathbf{S}}_{\mathbf{x}_{3}}\right)^{2}, \ldots,\left(\hat{\mathbf{S}}_{\mathbf{x}_{1}}+\cdots+\hat{\mathbf{S}}_{\mathbf{x}_{\ell}{ }_{-1}}\right)^{2}, \hat{\mathbf{S}}_{T}^{2}, \hat{S}_{T}^{3}$. In other words, the eigenvalues of $\left(\hat{\mathbf{S}}_{\mathbf{x}_{1}}+\hat{\mathbf{S}}_{\mathbf{x}_{2}}\right)^{2}$, $\left(\hat{\mathbf{S}}_{\mathbf{x}_{1}}+\hat{\mathbf{S}}_{\mathbf{x}_{2}}+\hat{\mathbf{S}}_{\mathbf{x}_{3}}\right)^{2}, \ldots,\left(\hat{\mathbf{S}}_{\mathbf{x}_{1}}+\cdots+\hat{\mathbf{S}}_{\mathbf{x}_{\ell^{3}-1}}\right)^{2}$ can be used as good quantum numbers for classifying the states of $\mathscr{H}_{S_{T}, S_{T}^{3}}$. Note that the operators associated with these quantum numbers are all scalars, i.e., they commute with the three components of $\hat{\mathbf{S}}_{T}$ : therefore, the eigenvectors of $H_{\Lambda_{1}}^{N}$ on $\mathscr{H}_{S_{T}, S_{T}^{3}}$ are invariant under the action of $\hat{\mathbf{S}}_{T}$, which implies in particular that $\operatorname{Tr}_{\mathscr{H}_{S_{T}, S_{T}^{3}}} \exp \left\{-\beta H_{\Lambda_{1}}^{N}\right\}$ is independent of $S_{T}^{3}$.
} 
where $\mathcal{H}_{\Lambda_{1}}^{N}$ is the bosonic hamiltonian in $\Lambda_{1}$ with Neumann boundary conditions (i.e., it is given in Eq. (1.7) with $\Lambda$ replaced by $\Lambda_{1}$ and the condition $\|\mathbf{x}-\mathbf{y}\|_{\Lambda}=1$ replaced by $\left.|\mathbf{x}-\mathbf{y}|=1\right)$. Inspired by Eqs. (1.7)-(1.8), we rewrite $\mathcal{H}_{\Lambda_{1}}^{N}=\mathcal{H}_{0, \Lambda_{1}}^{N}+\mathcal{K}_{\Lambda_{1}}^{N}$, with $\mathcal{H}_{0, \Lambda_{1}}^{N}=J S \sum_{\langle\mathbf{x}, \mathbf{y}\rangle \subset \Lambda_{1}}\left(a_{\mathbf{x}}^{\dagger}-a_{\mathbf{y}}^{\dagger}\right)\left(a_{\mathbf{x}}-a_{\mathbf{y}}\right)$ and $\mathcal{K}_{\Lambda_{1}}^{N}$ the interaction part, which can be bounded as follows (see Appendix $\mathrm{A}$ for a proof).

Proposition 2.3 (Estimate of $\mathcal{K}_{\Lambda_{1}}^{N}$ ).

There exists a finite constant $C$ such that

$$
\left|\mathcal{K}_{\Lambda_{1}}^{N}\right| \leq C \hat{N}_{\Lambda_{1}}^{2}
$$

where $\hat{N}_{\Lambda_{1}}:=\sum_{\mathbf{x} \in \Lambda_{1}} \hat{n}_{\mathbf{x}}$.

Using this estimate in (2.9) together with the fact that $\hat{N}_{\Lambda_{1}} \leq\left(S-S_{*}\right) \ell^{3}=(2 / c \tilde{\beta}) \ell^{5} \log (2 S+1)$, we get for a suitable constant $C^{\prime}$

$$
\tilde{\mathcal{Z}}\left(\beta, S, \Lambda_{1}\right) \leq\left(2 S \ell^{3}+1\right) e^{C^{\prime} \ell^{10} S^{-1} \log ^{2} S} \tilde{\mathcal{Z}}_{0}\left(\beta, S, \Lambda_{1}\right),
$$

where $\tilde{\mathcal{Z}}_{0}\left(\beta, S, \Lambda_{1}\right)$ stands for the partition functions of a free Bose gas in a box $\Lambda_{1}$ with free conditions at the boundary and constraint on the total number of particles $N_{\Lambda_{1}} \leq\left(S-S_{\star}\right) \ell^{3}$.

The estimate of $\tilde{\mathcal{Z}}_{0}$ is very simple: we rewrite the partition function in terms of the Neumann Fourier modes $\mathbf{k} \in \Lambda_{1, N}^{*}$ (see Appendix A) and drop the constraint on the total particle number except for the mode $\mathbf{k}=\mathbf{0}$ and obtain

$$
\tilde{\mathcal{Z}}_{0}\left(\beta, S, \Lambda_{1}\right) \leq \sum_{\substack{\left\{\tilde{n}_{\mathbf{k}}\right\}, \mathbf{k} \in \Lambda_{1, N}^{*} \\ \tilde{n}_{0} \leq\left(S-S_{\star}\right) \ell^{3}}} \exp \left\{-\tilde{\beta} J \sum_{\mathbf{k} \in \Lambda_{1, N}^{*}} \varepsilon(\mathbf{k}) \tilde{n}_{\mathbf{k}}\right\}=\left(S-S_{\star}\right) \ell^{3} \prod_{\substack{\mathbf{k} \in \Lambda_{1, N}^{*} \\ \mathbf{k} \neq 0}} \frac{1}{1-\exp \{-\tilde{\beta} \varepsilon(\mathbf{k})\}} .
$$

Putting together all the estimates, we obtain

$$
\frac{f(\beta, S, \Lambda)}{S} \geq \frac{1}{\ell^{3} \tilde{\beta}} \sum_{\mathbf{k} \neq \mathbf{0}} \log \left(1-e^{-\tilde{\beta} J \varepsilon(\mathbf{k})}\right)-\left(\text { const.) }\left[\frac{\ell^{7}(\log S)^{2}}{S}+\frac{\log \left(\ell^{3} S\right)}{\ell^{3}}\right] .\right.
$$

Finally, replacing the Riemann sum in the r.h.s. by the corresponding integral, we get

$$
\frac{f(\beta, S, \Lambda)}{S} \geq \frac{1}{\tilde{\beta}} \int_{\mathcal{B}} \frac{\mathrm{d} \mathbf{k}}{(2 \pi)^{3}} \log \left(1-e^{-\tilde{\beta} J \varepsilon(\mathbf{k})}\right)-(\text { const. })\left[\frac{\ell^{7}(\log S)^{2}}{S}+\frac{\log \left(\ell^{3} S\right)}{\ell^{3}}+\frac{1}{\ell}\right] .
$$

Optimizing over $\ell$ yields $\ell=S^{1 / 8}(\log S)^{-1 / 4}$ and the desired lower bound on the specific free energy.

\subsection{Upper Bound}

In this section we complete the proof of Theorem 1.1 by deriving a suitable (rough) upper bound to the free energy. The most relevant steps in the proof are an energy localization into Dirichlet boxes, the introduction of a fictitious magnetic field (which will be removed at the end) to take into account the constraint on the bosonic Hilbert space and the estimate proven in Proposition 2.3 on the interaction. A finer upper bound, supposedly optimal up to corrections of the order $1 / S$ included, will be presented elsewhere CGS.

Proposition 2.4 (Free energy upper bound).

Assume that $\beta=\tilde{\beta} S^{-1}$ as $S \rightarrow \infty$ for some $\tilde{\beta}>0$ constant. Then one has

$$
\frac{f(S, \beta)}{S} \leq \frac{1}{\tilde{\beta}} \int_{\mathcal{B}} \frac{\mathrm{d} \mathbf{k}}{(2 \pi)^{3}} \log \left(1-e^{-\tilde{\beta} J \varepsilon(\mathbf{k})}\right)+\mathcal{O}\left(S^{-1 / 6}(\log S)^{2 / 3}\right) .
$$


Proof. We start by adding an external magnetic field $h>0$ to the Hamiltonian: exploiting the positivity of $\hat{S}_{\mathbf{x}}^{z}+S$, one has the trivial inequality

$$
\mathcal{Z}(\beta, S, \Lambda) \geq \mathcal{Z}_{h}(\beta, S, \Lambda):=\operatorname{Tr}_{\mathscr{H}_{\Lambda}}\left(\exp \left\{-\beta H_{h}\right\}\right)
$$

for any $h>0$, where $H_{h}=H+h \sum_{\mathbf{x} \in \Lambda}\left(\hat{S}_{\mathbf{x}}^{3}+S\right)$. We fix $\ell \in \mathbb{N}$ and define the corridor $\mathcal{C}$ of width 1 as the minimal connected set on $\Lambda$ that contains $\left\{\mathbf{x} \in \Lambda: \mathbf{x}=\mathbf{n} \ell, \mathbf{n} \in \mathbb{Z}^{3}\right\}$ (here we assume for simplicity that $L$ is divisible by $\ell$ ). Note that $\Lambda \backslash \mathcal{C}$ is a union of boxes $\Lambda_{i}$ of side length $\ell-1$. We localize the energy in the boxes $\Lambda_{i}$ with Dirichlet boundary conditions, by proceeding as follows. Let us denote by $\mathscr{H}_{\mathcal{C}}$ and $\mathscr{H}_{\Lambda \backslash \mathcal{C}}$ the Hilbert spaces generated by the spins in $\mathcal{C}$ and $\Lambda \backslash \mathcal{C}$, respectively. Note that, if $\left|\downarrow_{\mathcal{C}}\right\rangle$ is the state of $\mathscr{H}_{\mathcal{C}}$ such that $S_{\mathbf{x}}^{3}=-S, \forall \mathbf{x} \in \mathcal{C}$, and $|\psi\rangle$ is a generic state of $\mathscr{H}_{\Lambda \backslash \mathcal{C}}$, then $\left(\left\langle\downarrow_{\mathcal{C}}|\otimes\langle\psi|) H_{h}\left(\left|\downarrow_{\mathcal{C}}\right\rangle \otimes|\psi\rangle\right)=\left\langle\psi\left|\sum_{i} H_{h, \Lambda_{i}}^{D}\right| \psi\right\rangle\right.\right.$, where

$$
H_{h, \Lambda_{i}}^{D}=H_{\Lambda_{i}}^{N}+h \sum_{\mathbf{x} \in \Lambda_{i}}\left(\hat{S}_{\mathbf{x}}^{3}+S\right)+J \sum_{\mathbf{x} \in \partial \Lambda_{i}}\left(S^{2}+S \hat{S}_{\mathbf{x}}^{3}\right)
$$

and the first sum runs over pairs of nearest neighbor sites, both belonging to $\Lambda_{i}$, while the second term is the boundary contribution, which should be thought of as an interaction term between the sites at the boundary of $\Lambda_{i}$ with the neighboring sites in the corridor $\mathcal{C}$. Obviously, the Hamiltonians $H_{\Lambda_{i}}^{D}$ all commute among each other and can be naturally thought as operators on $\mathscr{H}_{\Lambda_{i}}$. We also denote by $E_{i}^{D}$ and $\left|E_{i}^{D}\right\rangle$ the eigenvalues and eigenvectors of $H_{\Lambda_{i}}^{D}$ and set $\left|\left\{E_{i}^{D}\right\}\right\rangle:=\bigotimes_{i=1}^{L^{3} / \ell^{3}}\left|E_{i}^{D}\right\rangle$. Given these definitions, we note that

$$
\begin{aligned}
\mathcal{Z}_{h}(\beta, S, \Lambda) & \geq \sum_{\left\{E_{i}^{D}\right\}}\left(\left\langle\downarrow_{\mathcal{C}}\left|\otimes\left\langle\left\{E_{i}^{D}\right\}\right|\right) e^{-\beta H_{h}}\left(\left|\downarrow_{\mathcal{C}}\right\rangle \otimes\left|\left\{E_{i}^{D}\right\}\right\rangle\right)\right.\right. \\
& \geq \sum_{\left\{E_{i}^{D}\right\}} \exp \left\{-\beta\left(\left\langle\downarrow_{\mathcal{C}}\left|\otimes\left\langle\left\{E_{i}^{D}\right\}\right|\right) H_{h}\left(\left|\downarrow_{\mathcal{C}}\right\rangle \otimes\left|\left\{E_{i}^{D}\right\}\right\rangle\right)\right\}\right.\right. \\
& =\prod_{i=1}^{L^{3} / \ell^{3}} \sum_{E_{i}^{D}} e^{-\beta E_{i}^{D}}=:\left(\mathcal{Z}_{h}^{D}\left(\beta, S, \Lambda_{1}\right)\right)^{L^{3} / \ell^{3}},
\end{aligned}
$$

where, in order to go from the first to the second line, we used Jensen inequality to lift the expectation value to the exponent, while to go from the second to the third we used that the expression in braces is equal to $-\beta\left\langle\left\{E_{i}^{D}\right\}\left|\sum_{i} H_{\Lambda_{i}}^{D}\right|\left\{E_{i}^{D}\right\}\right\rangle$.

Now we use the bosonic representation, drop from the partition function $\mathcal{Z}_{h}^{D}\left(\beta, S, \Lambda_{1}\right)$ the contribution from large total occupation number $N_{\Lambda_{1}}$, estimate the interaction $\mathcal{K}_{\Lambda_{1}}^{D}$ and finally restore the missing part of the partition function. To this purpose we introduce a new parameter $\bar{N} \ll S$, which is going to be chosen later and notice that the bosonic analogue of $H_{h, \Lambda_{1}}^{D}$ is $\mathcal{H}_{h, \Lambda_{1}}^{D}=\mathcal{H}_{\Lambda_{1}}^{N}+h \sum_{\mathbf{x} \in \Lambda_{1}} n_{\mathbf{x}}+S J \sum_{\mathbf{x} \in \partial \Lambda_{1}} n_{\mathbf{x}}$. Now, in analogy with the case of Neumann boundary conditions, we can rewrite $\mathcal{H}_{h, \Lambda_{1}}^{D}=\mathcal{H}_{0, h, \Lambda_{1}}^{D}+\mathcal{K}_{h, \Lambda_{1}}^{D}$, where $\mathcal{H}_{0, h, \Lambda_{1}}^{D}$ stands for the quadratic part of $\mathcal{H}_{h, \Lambda_{1}}^{D}$, while $\mathcal{K}_{h, \Lambda_{1}}^{D}$ is at least quartic in the Bose operators and can be bounded exactly as in Eq.(2.10). Therefore,

$$
\mathcal{Z}_{h}^{D}\left(\beta, S, \Lambda_{1}\right) \geq \exp \left\{-\frac{C \tilde{\beta} \bar{N}^{2}}{S}\right\} \operatorname{Tr}_{\mathscr{F}_{\Lambda_{1}}^{0}}\left[\mathbb{1}\left(\sum_{\mathbf{x} \in \Lambda_{1}} n_{\mathbf{x}} \leq \bar{N}\right) \exp \left\{-\beta \mathcal{H}_{0, h, \Lambda_{1}}^{D}\right\}\right]
$$

where $\mathscr{F}_{\Lambda_{1}}^{0}$ is the unconstrained bosonic Hilbert space on $\Lambda_{1}$, i.e. the Bose particle number $n_{\mathbf{x}}$ is unbounded on $\mathscr{F}_{\Lambda_{1}}^{0}$ : note that the removal of the constraint is irrelevant, because $\bar{N} \ll S$ and, therefore, the condition that $n_{\mathbf{x}} \leq 2 S$ is automatically satisfied. Now we rewrite $\mathbb{1}\left(\sum_{\mathbf{x} \in \Lambda_{1}} n_{\mathbf{x}} \leq \bar{N}\right)=$ 
$1-\mathbb{1}\left(\sum_{\mathbf{x} \in \Lambda_{1}} n_{\mathbf{x}}>\bar{N}\right)$ and bound the contribution from the states with more than $\bar{N}$ particles as

$$
\begin{aligned}
& \sum_{\substack{\left\{n_{\mathbf{x}}\right\}, \mathbf{x} \in \Lambda_{1} \\
\sum n_{\mathbf{x}}>\bar{N}}}\left(\left\{n_{\mathbf{x}}\right\}\left|\exp \left\{-\beta \mathcal{H}_{0, h, \Lambda_{1}}^{D}\right\}\right|\left\{n_{\mathbf{x}}\right\}\right) \leq \sum_{\substack{\left\{n_{\mathbf{x}}\right\}, \mathbf{x} \in \Lambda_{1} \\
\sum n_{\mathbf{x}}>\bar{N}}}\left(\left\{n_{\mathbf{x}}\right\}\left|\exp \left\{-\tilde{\beta} S^{-1} h \sum_{\mathbf{x} \in \Lambda_{1}} \hat{n}_{\mathbf{x}}\right\}\right|\left\{n_{\mathbf{x}}\right\}\right) \\
& \leq e^{-\frac{1}{2} \tilde{\beta} S^{-1} h \bar{N}} \prod_{\mathbf{x} \in \Lambda_{1}} \sum_{n_{\mathbf{x}} \geq 0} e^{-\tilde{\beta} S^{-1} h n_{\mathbf{x}}} \leq e^{-\frac{1}{4} \tilde{\beta} S^{-1} h \bar{N}}
\end{aligned}
$$

provided that $h / S \ll 1$ and $S^{-1} h \bar{N} \gg \ell^{3} \log (S / h)$. The estimate (2.18) thus becomes

$$
\mathcal{Z}_{h}^{D}\left(\beta, S, \Lambda_{1}\right) \geq e^{-C \tilde{\beta} \bar{N}^{2} / S}\left(1-e^{-\frac{1}{4} \tilde{\beta} S^{-1} h \bar{N}}\right) \operatorname{Tr}_{\mathscr{F}_{\Lambda_{1}}^{0}}\left(\exp \left\{-\beta \mathcal{H}_{0, h, \Lambda_{1}}^{D}\right\}\right)
$$

In order to compute $\operatorname{Tr}_{\mathscr{F}_{\Lambda_{1}}}\left(\exp \left\{-\beta \mathcal{H}_{0, h, \Lambda_{1}}^{D}\right\}\right)$ we go to Fourier space: assuming for definiteness that $\Lambda_{1}=\left\{\mathbf{x} \in \mathbb{Z}^{3}: x_{i}=1, \ldots, \ell-1\right\}$, we rewrite $H_{0, h, \Lambda_{1}}^{D}$ by applying the Fourier transform $\tilde{a}_{\mathbf{k}}^{ \pm}=\sum_{\mathbf{x} \in \Lambda_{1}} \varphi_{\mathbf{k}}(\mathbf{x}) a_{\mathbf{x}}^{ \pm}$, where $\varphi_{\mathbf{k}}(\mathbf{x})=\prod_{i=1}^{3} \varphi_{k_{i}}\left(x_{i}\right), \varphi_{k}(x)=[2 /(\ell-1)]^{1 / 2} \sin (k x)$ and the set of momenta $\Lambda_{1, D}^{*}$ is $\Lambda_{1, D}^{*}=\left\{\frac{\pi}{\ell} \mathbf{n}, \mathbf{n} \in \mathbb{Z}^{3}: n_{i}=1, \ldots, \ell-1\right\}$. After Fourier transform we get

$$
\mathcal{H}_{0, h, \Lambda_{1}}^{D}=S J \sum_{\mathbf{k} \in \Lambda_{1, D}^{*}}\left(\varepsilon(\mathbf{k})+\frac{h}{S J}\right) \tilde{a}_{\mathbf{k}}^{\dagger} \tilde{a}_{\mathbf{k}},
$$

so that

$$
\operatorname{Tr}_{\mathscr{F}_{\Lambda_{1}}^{0}}\left(\exp \left\{-\beta \mathcal{H}_{0, h, \Lambda_{1}}^{D}\right\}\right)=\prod_{\mathbf{k} \in \Lambda_{1, D}^{*}} \frac{1}{1-e^{-\tilde{\beta} J(\varepsilon(\mathbf{k})+h / S J)}} .
$$

Putting all the estimates together gives

$$
\begin{aligned}
& -\frac{1}{\left|\Lambda_{1}\right|} \log \mathcal{Z}_{h}^{D}\left(\beta, S, \Lambda_{1}\right) \leq \\
& \leq \frac{1}{\ell^{3}} \sum_{\mathbf{k} \in \Lambda_{1}^{*}, \mathbf{k} \neq 0} \log \left(1-e^{-\tilde{\beta} J\left(\varepsilon(\mathbf{k})+\frac{h}{S J}\right)}\right)+(\text { const. })\left[\frac{\bar{N}^{2}}{\ell^{3} S}-\frac{1}{\ell^{3}} \log \left(1-e^{-\frac{1}{4} \tilde{\beta} S^{-1} h \bar{N}}\right)\right] \leq \\
& \leq \int_{\mathcal{B}} \frac{\mathrm{d} \mathbf{k}}{(2 \pi)^{3}} \log \left(1-e^{-\tilde{\beta} J \varepsilon(\mathbf{k})}\right)+(\text { const. })\left[\frac{\bar{N}^{2}}{\ell^{3} S}-\frac{1}{\ell^{3}} \log \left(1-e^{-\frac{1}{4} \tilde{\beta} S^{-1} h \bar{N}}\right)+\frac{h}{S}+\frac{1}{\ell}\right]
\end{aligned}
$$

To satisfy the condition $S^{-1} h \bar{N} \gg \ell^{3} \log (S / h)$ met during the proof we can pick $h=\bar{N}^{-1} S \ell^{3}(\log S)^{2}$. Optimizing over $\bar{N}$ and $\ell$ yields

$$
\bar{N}=C S^{2 / 3}(\log S)^{-2 / 3}, \quad \ell=C S^{1 / 6}(\log S)^{-2 / 3}, \quad h=C S^{5 / 6}(\log S)^{2 / 3} \ll S,
$$

for a suitable constant $C$. The corresponding relative error term in the energy is $\mathcal{O}\left(S^{-1 / 6} \log ^{2 / 3} S\right)$, which proves the proposition.

\section{A Two Technical Bounds}

In this appendix we prove Propositions 2.2 and 2.3 .

Proof of Proposition 2.2. Let us assume for definiteness that $\Lambda_{1}=\left\{\mathbf{x} \in \mathbb{Z}^{3}: x_{i}=1, \ldots, \ell\right\}$. We rewrite $H_{\Lambda_{1}}^{N}$ by applying the Fourier transform $\hat{\mathcal{S}}_{\mathbf{k}}=\sum_{\mathbf{x} \in \Lambda_{1}} \phi_{\mathbf{k}}(\mathbf{x}) \hat{\mathbf{S}}_{\mathbf{x}}$, where $\phi_{\mathbf{k}}(\mathbf{x})=\prod_{i=1}^{3} \phi_{k_{i}}\left(x_{i}\right)$,

$$
\phi_{k}(x)= \begin{cases}\ell^{-1 / 2}, & \text { if } k=0 \\ (2 / \ell)^{1 / 2} \cos \left(k\left(x-\frac{1}{2}\right)\right), & \text { if } k \neq 0\end{cases}
$$

and the set of momenta $\Lambda_{1, N}^{*}$ is

$$
\Lambda_{1, N}^{*}=\left\{\frac{\pi}{\ell} \mathbf{n}, \mathbf{n} \in \mathbb{Z}^{3}: n_{i}=0, \ldots, \ell-1\right\} .
$$


Then a straightforward computation shows that, if $\varepsilon(\mathbf{k})=\sum_{i=1}^{3}\left(1-\cos k_{i}\right)$,

$$
H_{\Lambda_{1}}^{N}=-3 S J\left(\ell^{3}-\ell^{2}\right)+J \sum_{\mathbf{k} \in \Lambda_{1, N}^{*}} \varepsilon(\mathbf{k}) \hat{\mathcal{S}}_{\mathbf{k}} \cdot \hat{\mathcal{S}}_{\mathbf{k}} .
$$

Now, for every $\mathbf{k} \neq \mathbf{0}$, one has $\varepsilon(\mathbf{k}) \geq c_{0} \ell^{-2}$, for a suitable $c_{0}>0$. Therefore,

$$
\left.H_{\Lambda_{1}}^{N}\right|_{\mathscr{H}_{S_{T}}} \geq-3 S J \ell^{3}+\left.J \frac{c_{0}}{\ell^{2}} \sum_{\mathbf{k} \neq \mathbf{0}} \hat{\mathcal{S}}_{\mathbf{k}} \cdot \hat{\mathcal{S}}_{\mathbf{k}}\right|_{\mathscr{H}_{S_{T}}}=-3 S J \ell^{3}+J \frac{c_{0}}{\ell^{2}}\left[S(S+1) \ell^{3}-\frac{S_{T}}{\ell^{3}}\left(S_{T}+1\right)\right]
$$

where in the last inequality we used Plancherel's identity $\sum_{\mathbf{k} \in \Lambda_{1, N}^{*}} \hat{\mathcal{S}}_{\mathbf{k}}^{2}=\sum_{\mathbf{x} \in \Lambda_{1}} \hat{\mathbf{S}}_{\mathbf{x}}^{2}=S(S+1) \ell^{3}$ and the definition of total spin operator $\hat{\mathbf{S}}_{T}=\ell^{3 / 2} \hat{\mathcal{S}}_{0}$, from which $\hat{\mathcal{S}}_{0}^{2}=\ell^{-3} S_{T}\left(S_{T}+1\right)$ on $\mathscr{H}_{S_{T}}$. Now, the expression in square brackets in the r.h.s. of Eq. A.4 can be bounded from below by $S \ell^{3}\left(S-S_{T} / \ell^{3}\right)$, so that

$$
\left.H_{\Lambda_{1}}^{N}\right|_{\mathscr{H}_{S_{T}}} \geq-3 S J \ell^{3}+c_{0} J S \ell\left(S-\frac{S_{T}}{\ell^{3}}\right)
$$

Therefore, for all $S_{T}$ such that $S-\ell^{-3} S_{T}>6 c_{0}^{-1} \ell^{2}$, we get $\left.H_{\Lambda_{1}}^{N}\right|_{\mathscr{H}_{S_{T}}} \geq \frac{c_{0}}{2} J \ell S\left(S-\ell^{-3} S_{T}\right)$, which proves the proposition with $c:=\frac{1}{2} J c_{0}$.

Proof of Proposition 2.3. We start by investigating the part of $\mathcal{K}_{\Lambda_{1}}^{N}$ associated with the square roots in the first term in (1.7). Using the fact that $A_{\mathbf{x}, \mathbf{y}}:=1-\sqrt{1-\frac{\hat{n}_{\mathbf{x}}}{2 S}} \sqrt{1-\frac{\hat{n}_{\mathbf{y}}}{2 S}}$ is a non-negative operator on the bosonic Hilbert space of interest, we get:

$$
\begin{aligned}
2 S J\left|\sum_{\langle\mathbf{x}, \mathbf{y}\rangle \subset \Lambda_{1}} a_{\mathbf{x}}^{\dagger} A_{\mathbf{x}, \mathbf{y}} a_{\mathbf{y}}\right| & =2 S J\left|\sum_{\langle\mathbf{x}, \mathbf{y}\rangle \subset \Lambda_{1}} a_{\mathbf{x}}^{\dagger} A_{\mathbf{x}, \mathbf{y}}^{1 / 2} A_{\mathbf{x}, \mathbf{y}}^{1 / 2} a_{\mathbf{y}}\right| \leq 2 S J \sum_{\langle\mathbf{x}, \mathbf{y}\rangle \subset \Lambda_{1}} a_{\mathbf{x}}^{\dagger} A_{\mathbf{x}, \mathbf{y}} a_{\mathbf{x}} \leq \\
& \left.\leq J \sum_{\langle\mathbf{x}, \mathbf{y}\rangle \subset \Lambda_{1}} a_{\mathbf{x}}^{\dagger}\left(n_{\mathbf{x}}+n_{\mathbf{y}}\right) a_{\mathbf{x}} \leq \text { (const. }\right)\left(\sum_{\mathbf{x} \in \Lambda_{1}} \hat{n}_{\mathbf{x}}\right)^{2}
\end{aligned}
$$

where in the first line we used the Cauchy-Schwarz inequality, while to go from the first to the second line we used the simple fact that $1-\sqrt{1-x} \leq x, \forall x \in[0,1]$. Finally, the remaining term in $\mathcal{K}_{\Lambda_{1}}^{N}$ can be bounded trivially as $J\left|\sum_{\langle\mathbf{x}, \mathbf{y}\rangle \subset \Lambda_{1}} n_{\mathbf{x}} n_{\mathbf{y}}\right| \leq$ (const.) $\left(\sum_{\mathbf{x} \in \Lambda_{1}} \hat{n}_{\mathbf{x}}\right)^{2}$, which implies the desired estimate.

Acknowledgements. We gratefully acknowledge financial support from the ERC Starting Grant CoMBoS-239694. We thank E.H. Lieb, B. Nachtergaele, R. Seiringer and J.-P. Solovej for useful comments and discussions.

\section{References}

[B1] F. BLoch, Zur Theorie des Ferromagnetismus, Z. Physik 61 (1930), 206-219.

[B2] F. BLoch, Zur Theorie des Austauschproblems und der Remanenzerscheinung der Ferromagnetika, Z. Physik 74 (1932), 295-335.

[BFLlS] J. Bricmont, J.-R. Fontaine, J.L. Lebowitz, E.H. Lieb, T. Spencer, Lattice Systems with a Continuous Symmetry III. Low Temperature Asymptotic Expansion for the Plane Rotator Model, Commun. Math. Phys. 78 (1981), 545-566.

[C-TDL] C. Cohen-Tannoudji, B. Diu, F. Lalö̈, Quantum Mechanics, Volume 2, Wiley, New York, 1977. 
[CS1] G.J. Conlon, J.P. SoloveJ, On Asymptotic Limits for the Quantum Heisenberg Model, J. Phys. A: Math. Gen. 23 (1990), 3199-3213.

[CS2] G.J. Conlon, J.P. SoloveJ, Upper Bound on the Free Energy of the Spin 1/2 Heisenberg Ferromagnet, Lett. Math. Phys. 23 (1991), 223-231.

[CS3] G.J. Conlon, J.P. Solovej, Uniform Convergence of the Free Energy of the Classical Heisenberg Model to That of the Gaussian Model, J. Stat. Phys. 65 (1991), 235-245.

[CH] J.F. Cooke, H.H. HAhn, Application of the Hard-Core Boson Formalism to the Heisenberg Ferromagnet, Phys. Rev. B 1 (1970), 1243-1250.

[CGS] M. Correggi, A. Giuliani, R. Seiringer, in preparation.

[D1] F.J. Dyson, General Theory of Spin-Waves Interactions, Phys. Rev. 102 (1956), 1217-1230.

[D2] F.J. Dyson, Thermodynamic Behavior of an Ideal Ferromagnet, Phys. Rev. 102 (1956), 1230-1244.

[DLS] F.J. Dyson, E.H. Lieb, B. Simon, Phase Transitions in the Quantum Heisenberg Model, Phys. Rev. Lett. 37 (1976), 120-123; Phase Transitions in Quantum Spin Systems with Isotropic and Nonisotropic Interactions, J. Stat. Phys. 18 (1978), 335-383.

[FSS] J. Fröhlich, B. Simon, T. Spencer, Infrared Bounds, Phase Transitions and Continuous Symmetry Breaking, Commun. Math. Phys. 50 (1976), 79-85.

[HK] C. Herring, C. Kittel, On the Theory of Spin Waves in Ferromagnetic Media, Phys. Rev. 81 (1951), 869-880.

[H1] C.P. Hofmann, Spontaneous magnetization of the $O(3)$ ferromagnet at low temperatures Phys. Rev. B 65 (2002), 094430.

[H2] C.P. Hofmann, Spontaneous magnetization of an ideal ferromagnet: Beyond Dyson's analysis, Phys. Rev. B 84 (2011), 064414.

[HP] T. Holstein, H. Primakoff, Field Dependence of the Intrinsic Domain Magnetization of a Ferromagnet, Phys. Rev. 58 (1940), 1098-1113.

[Ke] F. Keffer, in Handbuch der Physik 18/2, 1-273, Springer-Verlag, Berlin, 1966.

[KL] F. Keffer, R. Loudon, Simple Physical Theory of Spin Wave Interactions, J. Appl. Phys. (Suppl.) 32 (1961), 2S.

[Kr] H.A. Kramers, Commun. Kemerlingh Onnes Lab. Univ. Leiden 22, Suppl. No. 83 (1936).

[L] E.H. Lieb, The Classical Limit of Quantum Spin Systems, Commun. Math. Phys. 31 (1973), $327-340$.

[LS] E.H. Lieb, R. Seiringer, Proof of Bose-Einstein condensation for dilute trapped gases, Phys. Rev. Lett 88 (2002), 170409.

[LSY] E.H. Lieb, R. Seiringer, J. Yngvason, A rigorous derivation of the Gross-Pitaevskii energy functional, Phys. Rev. A 61 (2000), 043602.

[LSSY] E.H. Lieb, R. Seiringer, J.-P. Solovej, J. Yngvason, The Mathematics of the Bose Gas and its Condensation, Oberwolfach Seminars 34, Birkhäuser, Basel, 2005, also available at arXiv:cond-mat/0610117] [cond-mat.stat-mech].

[LY] E.H. Lieb, J. Yngvason, Ground state energy of the low density Bose gas, Phys. Rev. Lett. 80 (1998), 2504-2507.

[MN1] T. Michoel, B. Nachtergaele, The large-spin asymptotics of the ferromagnetic XXZ chain, Markov Process. Related Fields 11 (2005), 237-266.

[MN2] T. Michoel, B. Nachtergaele, Central limit theorems for the large-spin asymptotics of quantum spins, Prob. Theory Rel. Fields 130 (2004), 493-517.

[M] T. Morita, Bose-Einstein Lattice Gases Equivalent to the Heisenberg Model of Ferro-, Antiferro- and Ferri-Magnetism, Prog. Theor. Phys. 20 (1958), 614-624. 
[Og] T. Oguchi, Theory of Spin-Wave Interactions in Ferro- and Antiferromagnetism, Phys. Rev. 117 (1960), 117-123.

[Op] W. Opechowski, Über die temperaturabhängigkeit der magnetisierung eines ferromagnetikums bei tiefen temperaturen, Physica 4 (1937), 715-722.

[RL] E. Rastelli, P. A. Lindgard, Exact results for spin-wave renormalisation in Heisenberg, and planar ferromagnets, J. Phys. C 12 (1979), 1899-1916.

[Sc] M.R. Schafroth, Self-Consistent Spin-Wave Theory for the Ferromagnetic Exchange Problem, Proc. Phys. Soc. (London) A67 (1954), 33-38.

[Sz] J. SzAniECKI, On certain divergences in the quantum spin wave theory of ferromagnetism, J. Phys. C 7 (1974), 4113-4125.

[T] B. Тотн, Improved Lower Bound on the Thermodynamic Pressure of the Spin 1/2 Heisenberg Ferromagnet, Lett. Math. Phys. 28 (1993), 75-84.

[VLP] V.G. VAKs, A.I. Larkin, S.A. Pikin, Thermodynamics of an ideal ferromagnetic substance, Sov. Phys. JETP 26 (1968), 188-199.

[vHBW] J.L. van Hemmen, A.A.S. Brito, W.F. Wreszinski, Spin waves in quantum ferromagnets, J. Stat. Phys. 37 (1984), 187-213.

[VK] J. Van Kranendonk, Spin-deviation theory of ferromagnetism, I and II, Physica 21 (1955), 749-766 and 925-945.

[W] D.C. Wallace, Renormalized Spin Waves in the Heisenberg Ferromagnet, Phys. Rev. 153 (1967), 547-558.

[YW] D.H. YAng, Y. WANG, Green's-function diagrammatic technique for complicated level systems. II. An application to the spin-1 Heisenberg ferromagnet with easy-axis single-ion anisotropy, Phys. Rev. B 12 (1975), 1057-1070.

[Z] J. ZitTARTZ, On the spin wave problem in the Heisenberg model of Ferromagnetism, $Z$. Phys. 184 (1965), 506-520. 